\title{
Uneinheitliche Vermeidungskosten und Preissignale im Klimaschutz
}

Für den Schutz des globalen Klimas und zur Erreichung des $1,5^{\circ} \mathrm{C}$-Ziels müssen die zukünftigen Treibhausgasemissionen begrenzt werden. Wer, wo und zu welchem Zweck eine Tonne $\mathrm{CO}_{2}$ (oder eine entsprechende Menge eines anderen Treibhausgases) emittiert (wird), ist für die zukünftige Entwicklung des Klimas nicht relevant. Die $\mathrm{CO}_{2}$-Tonnen unterscheiden sich hinsichtlich des Klimaeffekts nicht voneinander und können insofern als homogenes Gut angesehen werden. Dennoch ist die Emission von Treibhausgasen nicht mit einem einheitlichen Preis verbunden, der bei vollkommenem Wettbewerb auf einem räumlich flexiblen Weltmarkt zu erwarten wäre.

Der Marktpreis für eine Emission ergibt sich bei feststehendem Angebot (Cap) aus den Grenzvermeidungskosten bzw. entgangenen Nutzen, die mit einer weiteren Tonne Emission realisiert werden können. Unterschiedliche Preise für das homogene Gut widersprechen dem "Law of one Price" (Jevons, 1888) und deuten auf Ineffizienzen und Marktunvollkommenheiten beim Klimaschutz hin (Bardt, 2018). Unterschiedliche Preissignale führen dazu, dass an einer Stelle höhere Vermeidungskosten bzw. Nutzeneinbußen in Kauf genommen werden, um eine Tonne Treibhausgasemissionen zu vermeiden, während dies an anderer Stelle mit geringeren Kosten möglich gewesen wäre. Die globalen Gesamtkosten des Klimaschutzes sind damit höher als bei einem effizienten Klimaschutz nötig.

Die Preisdifferenzen entstehen aus einer Kombination uneinheitlicher Angebots- und Nachfragebedingungen. Das Angebot an Emissionsrechten wird durch die jeweiligen nationalen Klimaschutzregeln definiert (oder das Fehlen entsprechender Grenzen), die Nachfrage bestimmt sich durch technologische Gegebenheiten und die jeweilige Wirtschaftsstruktur, aus der sich Vermeidungskosten bzw. mit den Emissionen verbundene Nutzengrößen ergeben.

Zu differenzieren sind Kostenunterschiede zwischen Sektoren, die auf differenzierten Technologiekosten beruhen, und Kostenunterschiede zwischen Ländern, die darauf zurückzuführen sind, dass die aktuellen und vergangenen

(C) Der/die Autor:in(nen) 2021. Open Access: Dieser Artikel wird unter der Creative Commons Namensnennung 4.0 International Lizenz veröffentlicht (creativecommons.org/licenses/by/4.0/deed.de).

Open Access wird durch die ZBW - Leibniz-Informationszentrum Wirtschaft gefördert.
Klimaschutzniveaus uneinheitlich sind. Dort, wo günstige Vermeidungspotenziale bereits realisiert wurden, ist mit höheren Kosten zu rechnen. Eine Vereinheitlichung würde die Chance mit sich bringen, globalen Klimaschutz mit geringstmöglichen Kosten zu verwirklichen.

\section{Sektorale Kosten- und Preisunterschiede}

Die europäische und deutsche Klimapolitik hat für die verschiedenen Sektoren jeweils spezifische Ziele vorgesehen. Daraus ergeben sich stark differenzierende Preissignale. Besonders hoch waren die impliziten $\mathrm{CO}_{2}-$ Kosten für Pkw, da Hersteller bei der Überschreitung der vorgesehenen Flottengrenzwerte hohe Strafzahlungen leisten müssen. Diese liegen bei 95 Euro je Gramm Überschreitung und Fahrzeug. Bei einer unterstellten Gesamtlaufleistung eines Fahrzeugs von 200.000 km beläuft sich der implizite $\mathrm{CO}_{2}$-Preis auf 475 Euro. Der Preis für eine Tonne $\mathrm{CO}_{2}$ im europäischen Emissionshandel ist hingegen 2021 erstmals auf über 40 Euro gestiegen. Schon allein aus dieser Relation wird deutlich, dass für die Vermeidung von Emissionen in der Fahrzeugherstellung (hinzu kommen die expliziten und impliziten $\mathrm{CO}_{2}$-Kosten der Kraftstoffbesteuerung sowie die Förderung von Elektrofahrzeugen) deutlich teurere Maßnahmen ergriffen werden als in anderen Bereichen.

McKinsey (2020) rechnet für 2030 mit weiterhin stark differenzierten sektoralen Vermeidungskosten in Europa. Für wichtige Industrieprozesse werden diese 2030 bei einem Wert von 50 bis 100 Euro je Tonne gesehen. Bis 2050 steigen sie noch einmal spürbar an und liegen im Wesentlichen im dreistelligen Bereich. Klimaschutz im Gebäudebereich wird mit vielen Maßnahmen hingegen im negativen Kostenbereich gesehen, d.h. hier werden höhere Einsparungen als Zusatzkosten unterstellt. Dies gilt insbesondere für solarthermisches Heizen mit negativen Vermeidungskosten von unter -400 Euro je Tonne. Aber

Dr. Hubertus Bardt leitet den Bereich Wissenschaft am IW in Köln und ist Lehrbeauftragter an der Heinrich-Heine-Universität Düsseldorf. 
auch bei der Hausisolierung wird mit Einsparpotenzialen gerechnet. Überraschend ist, dass für die Elektromobilität negative Vermeidungskosten unterstellt werden. Dieser Effekt basiert auf der Annahme deutlich sinkender Preise für Elektroautos durch Preissenkungen für Batterien. $\mathrm{Ob}$ dies angesichts der hohen Rohstoffkostenanteile der Batterien wirklich möglich ist, wenn eine deutlich steigende Rohstoffnachfrage für Batterien auftritt, kann jedoch angezweifelt werden.

Die ökonomisch naheliegende Konsequenz dieser erheblichen Preisunterschiede läge darin, die staatlich gesetzten Preissignale zu vereinheitlichen und über die Marktpreismechanismen zu einem kosteneffizienten Einsatz von Klimaschutzmaßnahmen zu kommen. Über ein einheitliches preissetzendes und mengenbegrenzendes Instrument wie den Emissionshandel könnte das vorgegebene Emissionsziel zu geringstmöglichen Kosten erreicht werden. Unterschiedliche Positionen auf der Lernkurve, Entwicklungsnotwendigkeiten, Pfadabhängigkeiten oder Infrastrukturvoraussetzungen würden zusätzliche sektorspezifische Maßnahmen erfordern, die das zentrale, sektorübergreifende Steuerungsinstrument und das einheitliche Preissignal ergänzen.

Das Konzept des sektorübergreifenden Einheitspreises kommt jedoch an Grenzen, da die realwirtschaftlichen Auswirkungen in einer Welt ohne vergleichbare internationale Preissignale zusätzliche und sehr uneinheitliche Kosten verursachen. Ein einheitlicher $\mathrm{CO}_{2}$-Preis für Verkehr und Industrie hätte beispielsweise zur Folge, dass die Anreize verschwinden, hohe Kosten zur Verringerung der Klimawirkungen des Straßenverkehrs zu tragen. Die geringeren Emissionsreduktionen des Verkehrs müssten dann von anderen Sektoren wie der Industrie getragen werden. Höhere $\mathrm{CO}_{2}$-Kosten würden jedoch die Wettbewerbsfähigkeiten der energie- bzw. emissionsintensiven Industrien beeinträchtigen und damit das Carbon-Leakage-Risiko erhöhen. Höhere Kosten, die im internationalen Wettbewerb nicht an Kunden weitergegeben werden können, erhöhen den Druck zur Kostenreduktion, durch erwünschte Effizienzsteigerung oder unerwünschte Verschiebung von Investitionen und letztlich Produktion an Standorten mit niedrigeren $\mathrm{CO}_{2}$-Kosten.

Während Sektoren und Industrien ohne internationalen Wettbewerb ihre Kosten an die Verbraucher:innen weitergeben können, ist dies in Bereichen mit starken realen oder potenziellen Import- oder Exportanteilen nicht möglich. Die Industrie gehört typischerweise zu letzteren, Energiewirtschaft und landgestützter Verkehr sind ersteren zuzurechnen. Hier sind zudem deutlich geringere Preiselastizitäten festzustellen, Verbraucher:innen haben also eine höhere Bereitschaft, diese Kosten auch zu tragen.
Abbildung 1

Anteil der preislichen Belastung an globalen

Emissionen

Ende 2020

in US-\$

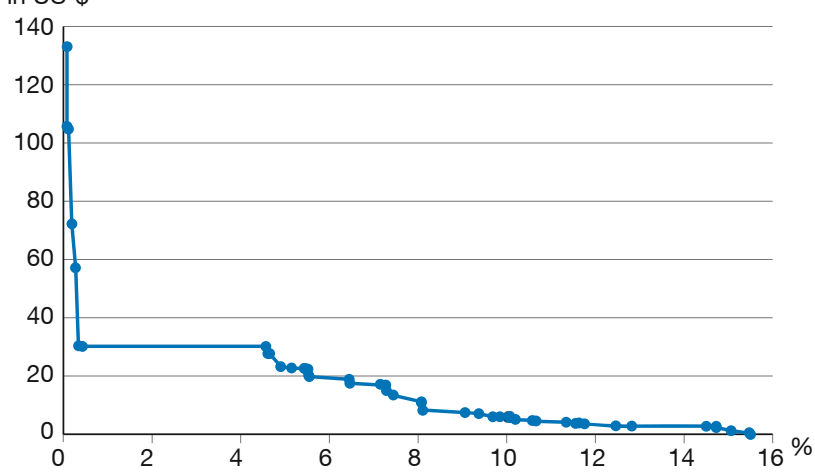

Quelle: Weltbank.

Die einfache ökonomische Lösung eines einheitlichen sektorübergreifenden Preises als alleiniges Klimaschutzinstrument führt bei unterschiedlichen internationalen Preisen für Treibhausgasemissionen nicht zur idealtypischen Lösung eines effizienten Klimaschutzes. Vielmehr müssen die Wohlstandsverluste durch Wettbewerbsverschlechterungen für energieintensive Branchen berücksichtigt und überwunden werden, ohne dass die erwünschten Anreize zur Treibhausgasreduktion unwirksam werden. Fördermaßnahmen für klimafreundliche Investitionen können ein Weg sein, die gewünschten Modernisierungsinvestitionen im Inland möglich zu machen und damit die Basis für zukünftige klimafreundliche Produktion und Beschäftigung am heimischen Standort zu legen.

\section{Internationale Kosten- und Preisunterschiede}

Auch international sind erhebliche Kostenunterschiede zu verzeichnen, die für einen effizienten globalen Klimaschutz problematisch sind und nationale Regelungen erschweren. Gerade einmal ein Sechstel der globalen Emissionen unterliegen einem expliziten Preis durch eine $\mathrm{CO}_{2}$-Steuer oder ein Emissionshandelssystem. Bei weniger als $1 \%$ der Emissionen ist der angelegte Preis höher als im EU-Emissionshandel, der mit gut $4 \%$ der globalen Emissionen und einem Preis oberhalb der 30US-\$-Linie das weltweit umfassendste Preissignal für Treibhausgasemissionen aussendet (vgl. Abbildung 1). Über $90 \%$ der Emissionen sind mit weniger als 10 US-\$ pro Tonne bepreist, über $80 \%$ sind ohne explizites Preissignal. Am anderen Ende des Spektrums liegen nationale Steuern in den skandinavischen Ländern, Liechtenstein und der Schweiz.

Auch wenn andere emissionsbegrenzende Regulierungen und implizite Preise an dieser Stelle nicht berücksichtigt 
werden, ergeben sich daraus gravierende Konsequenzen. So werden Emissionen innerhalb bestimmter Preissysteme eher reduziert als solche, bei denen der Ausstoß für den Emittenten keine Kosten verursacht. Die globale Klimaschutzaufgabe würde zu günstigeren Gesamtkosten erreicht werden, wenn auch tatsächlich weltweit die preiswertesten Klimaschutzpotenziale genutzt werden können.

Im Rahmen des Kyoto-Abkommens konnten preisgünstigere Vermeidungsmaßnahmen in Drittländern mit realisiert und auf die Emissionsverpflichtungen im Rahmen des europäischen Emissionshandels angerechnet werden. Diese Maßnahmen sind aufgrund von Zweifeln, ob bestimmte Vermeidungsmaßnahmen wirklich zusätzlich getätigt wurden, in ein kritisches Licht geraten. Der Ansatz, dass günstigere Klimaschutzoptionen zunächst genutzt werden sollten, ist jedoch weiterhin effizienzsteigernd. Aktuelle Preise für daraus resultierende Emissionsgutschriften liegen nahe der Nullinie, würden sich bei steigender Nachfrage je nach Einrechnung früherer Projekte aber wieder nach oben entwickeln (DEHSt, 2018).

Die Nutzung internationaler Potenziale hat aber nicht nur direkte Folgen für die kostenminimale Verfolgung kurzfristiger Klimaschutzziele und ist insofern vorteilhaft für die anspruchsvollen Klimaschutz verfolgenden Industrieländer. Für die Zielländer bedeutet dies, dass Investitionen in klimafreundliche Technologien vorgenommen werden, die gleichzeitig ökologisch vorteilhaft sind und die wirtschaftliche Modernisierung unterstützen. Damit werden auch die günstigsten heimischen Potenziale genutzt, sodass weitere inländische Klimaschutzbemühungen für diese Länder teurer werden.

Wie günstig die Reduktion von Treibhausgasen gemessen an den nationalen Zielen ist, zeigt auch das chinesische Beispiel. Unter dem Kyoto-Abkommen war China führend in der Implementierung von CDM-Projekten (Clean Development Mechanism), mit denen beispielsweise deutsche Energieversorger zusätzliche Emissionsrechte für die Anrechnung in Europa schaffen konnten. Die damaligen Kostenvorteile bestehen auch mit Blick auf den neu ins Leben gerufenen chinesischen Emissionshandel fort. Hier wird mit Preisen für die Emission von unter 10 Euro je Tonne gerechnet (China Carbon Forum, 2020), was deutlich unter dem europäischen Preisniveau liegt.

Die differenzierenden Kosten-, Anspruchs- und Preisniveaus können nachteilige Wirkungen auf die Wettbewerbssituation der besonders betroffenen Industrien in Ländern mit einer höheren Bepreisung haben. Dies wird über unterschiedliche Carbon-Leakage-Regeln, Ausgleichsmechanismen, Förderansätze oder möglicherweise über Grenzabgaben ausgeglichen (aus dem
Moore et al., 2019; Koch und Bassa Mama, 2019). Damit werden zahlreiche letztlich diskretionäre Eingriffe in Wettbewerbsprozesse notwendig, die sogar überkompensierend und damit strukturkonservierend eingesetzt werden können. Ein stiller Wettbewerb um die sektoral niedrigsten $\mathrm{CO}_{2}$-Kosten in den Branchen mit den größten Emissionen wäre aber kaum vereinbar mit den international vereinbarten Bemühungen um das globale Gut Klimaschutz (Bardt, 2005).

Derartige Ausnahmen bedürfen somit einer internationalen Koordination. Dies gilt auch für die Frage des Grenzausgleichs, der sowohl in Europa wie den USA diskutiert wird. Dieser birgt neben seiner Schutzwirkung und der Gefahr, dass er protektionistisch ausgestaltet wird, das Risiko weiterer Handelskonflikte, wie bereits der Versuch der EU ausgelöst hatte, den internationalen Flugverkehr mit in den europäischen Emissionshandel zu integrieren (Kolev et al., 2021; Wissenschaftlicher Beirat, 2021).

\section{Konsequenzen}

Der nationale Klimaschutz stößt - unter anderem - dort an Grenzen, wo er mit absehbaren Wettbewerbsnachteilen verbunden ist und wo ein Abwägungsprozess zwischen einem Beitrag zum globalen Klimaschutz und einem eigenen Wohlstandsverlust vorgenommen werden muss. Das offensichtliche Problem eines fehlenden globalen Rahmens mit einheitlichen Anspruchsniveaus im Klimaschutz ist absehbar nicht zu lösen. Auch sektorspezifische Abkommen, die den $\mathrm{CO}_{2}$-Preis und damit den Klimaschutz als differenzierenden Wettbewerbsfaktor eliminieren, sind nicht in greifbarer Nähe. Vor diesem Hintergrund erscheinen zwei Ansätze vielversprechend:

1. Preiswerte Klimaschutztechnologie: Der befürchtete Trade-off entsteht nur dann, wenn klimaschützende Technologien ohne eine Bepreisung von Emissionen teurer sind als traditionelle Verfahren. Wenn die technologischen Vermeidungskosten negativ sind und auch sonst keine gravierenden Nachteile in Kauf genommen werden müssen, können sich die Technologien auch ohne globale Absprachen durchsetzen. Die erneuerbaren Energien können hierfür als Beispiel dienen. Zwar ist die eingeschränkte Speicherfähigkeit weiterhin ein Problem, dennoch konnten die Stromentstehungskosten soweit gesenkt werden, dass vielfach eine Wettbewerbsfähigkeit besteht oder es sich bei den erneuerbaren sogar um die günstigste Form der Strom- und Wärmeerzeugung handelt. Die Rolle der vorangehenden und wirtschaftlich stärksten Industrieländer liegt dann nicht so sehr im heimischen Klimaschutz, sondern in der Finanzierung der Entwicklung und der Lernkurven dieser Technologien, 
wodurch ein weitaus größerer Klimaschutzeffekt erzielt werden kann.

2. Marktmechanismus etablieren: Um die Produktion von energieintensiven Gütern nicht nur aufgrund der unterschiedlichen Anspruchs- und Preisniveaus international schrittweise verlagern zu müssen, bleibt ein Ausgleich unterschiedlicher Klimaschutzkostenbelastungen notwendig. Ein wichtiger Beitrag kann darin liegen, internationale Klimaschutzprojekte wieder nutzbar zu machen. Auch wenn ein vollständiger Preisausgleich eine uneingeschränkte und damit unrealistische Anrechnung erfordern würde, könnte ein Handel von Emissionsrechten in verschiedenen Weltregionen den besonders betroffenen Branchen helfen, ihren Klimafußabdruck zu verringern und dennoch weiter zu wettbewerbsfähigen Kosten zu produzieren. Ein solcher Mechanismus ist in Artikel 6 des Pariser Klimaschutzabkommens vorgesehen, bisher aber noch nicht umgesetzt worden.

\section{Literatur}

aus dem Moore, N., P. Großkurth und M. Themann (2019), Multinational corporations and the EU Emissions Trading System: The specter of asset erosion and creeping Deindustrialization, Journal of Environmental Economics and Management, 94, 1-26.

Bardt, H. (2005), Klimaschutz und Anpassung: Merkmale unterschiedlicher Politikstrategien, Vierteljahrshefte zur Wirtschaftsforschung, 74(2), 259-269.

Bardt, H. (2018), Law of one price - Klimapolitik zwischen Allokation und Verteilung, ORDO - Jahrbuch für die Ordnung von Wirtschaft und Gesellschaft, 68, 303-321.

China Carbon Forum (2020), China Carbon Pricing Survey.

DEHSt - Deutsche Emissionshandelsstelle (2018), Marginal cost of CER supply and implications of demand sources.

Jevons, W. S. (1888), The Theory of Political Economy.

Koch, N., H. Bassa Mama (2019), Does the EU Emissions Trading System induce investment leakage? Evidence from German multinational firms, Energy Economics, 81, 479-492.

Kolev, G., R. Kube, T. Schaefer und L. Stolle (2021), Carbon Border Adjustment Mechanism (CBAM), Motivation, Ausgestaltung und wirtschaftliche Implikationen eines $\mathrm{CO}_{2}$-Grenzausgleichs in der EU, IWPolicy Paper, 6.

McKinsey (2020), Net-Zero Europe - Decarbonization pathways and socioeconomic implications.

Wissenschaftlicher Beirat beim Bundesministerium für Wirtschaft und Energie (2021), Ein $\mathrm{CO}_{2}$-Grenzausgleich als Baustein eines Klimaclubs. 